\title{
Effectiveness of ursodeoxycholic acid therapy in intrahepatic cholestatsis of pregnancy
}

\author{
Devdatt I. Pitale ${ }^{1 *}$, Sujata N. Jadhav ${ }^{2}$
}

\begin{abstract}
${ }^{1}$ Department of Obstetrics and Gynecology, INHS Sandhani, Mumbai, Maharashtra, India
${ }^{2}$ Department of Obstetrics and Gynecology, MH Dehradun, Uttarakhand, India
\end{abstract}

Received: 14 December 2019

Accepted: 06 January 2020

\author{
*Correspondence: \\ Dr. Devdatt I. Pitale, \\ E-mail: dipu.pitale@gmail.com
}

Copyright: () the author(s), publisher and licensee Medip Academy. This is an open-access article distributed under the terms of the Creative Commons Attribution Non-Commercial License, which permits unrestricted non-commercial use, distribution, and reproduction in any medium, provided the original work is properly cited.

\begin{abstract}
Background: Intrahepatic cholestasis of pregnancy (ICP) is a pregnancy specific disorder and commonly presents with maternal pruritus, abnormal liver function tests and adverse maternal -foetal outcomes. ICP lacks protocol-based therapy as etiology is multifactorial and is based on symptomatic treatment. The overall incidence of ICP is variable from 0.1 to $15.6 \%$ worldwide. The aim of this study is to assess the effectiveness of UDCA in ICP with regards to reduction in pruritus, normalizing LFTs and maternal-foetal outcomes.

Methods: This multicentric prospective study was performed from June 2017 to December 2019 in pregnant women with ICP attending the Antenatal clinic at INHS Patanjali, MH Dehradun, INHS Asvini, INHS Sandhani. In this study, 50 women with ICP. who satisfied the inclusion and exclusion criteria were started on UDCA therapy. The effectiveness of therapy was evaluated on the basis of normalization of LFT levels, reduction in pruritus, safe confinement, maternal-foetal outcomes and adverse effects if any

Results: The pregnant women with ICP on UDCA therapy showed marked improvement in pruritus, near normal LFT levels. After the UDCA therapy the frequency and intensity of pruritus was reduced in $50(100 \%)$ of women. Safe confinement was achieved, with normal delivery in $45(90 \%)$ women with no any major adverse effects and adverse maternal-foetal outcomes.

Conclusions: This study shows the effectiveness of URCA therapy in reducing the ICP associated pruritus, normalizing LFTs and safe confinement without any major adverse effects. UDCA therapy is an effective and safe option in ICP.
\end{abstract}

Keywords: Intrahepatic cholestasis, Pregnancy, Ursodeoxycholic acid

\section{INTRODUCTION}

Intrahepatic cholestasis of pregnancy (ICP) is a common pregnancy-specific disorder, most commonly occurring in the late second or third trimesters of pregnancy. ICP clinically presents with maternal pruritus (especially over palms and soles and at night) altered liver function tests (elevated serum transaminases). ${ }^{1}$ The overall incidence of ICP is variable from 0.1 to $15.6 \%$ worldwide..$^{2,3}$ ICP has a multifactorial etio-pathogenesis related to sex hormone synthesis, environmental factors, and genetic predisposition. ${ }^{4,5} \mathrm{CP}$ is associated with adverse foetal outcomes like preterm delivery, neonatal respiratory distress syndrome, fetal distress, and sudden intrauterine fetal death along with increased incidence of postpartum hemorrhage. ${ }^{6,7}$

ICP is a high-risk pregnancy and the delivery should be timed properly, outweighing the risk of prematurity and ICP complications. Induction of labour is recommended in such women with ICP after 37 weeks of gestation. ${ }^{7}$ The cesarean section rate is not increased by the early 
induction of labor Glantz et al, reported an increased risk of spontaneous preterm labour, asphyxial events, meconium stained liquor. ${ }^{8,9}$

ICP lacks protocol-based therapy and treatments are focused on symptomatic relief. Cholestyramine, SAdenosyl-L-methionine (SAMe), and ursodeoxycholic acid have been used. ${ }^{10-12}$ Studies have shown UDCA to be effective in reducing pruritus and normalising the liver function tests in ICP. ${ }^{13}$

The aim of this study is to assess the effectiveness of UDCA in ICP with regards to reduction in pruritus, normalizing LFTs and maternal-foetal outcomes.

\section{METHODS}

The prospective multicentric observational study was performed from June 2017 to December 2019 in pregnant women with ICP attending the antenatal clinic at INHS Patanjali, MH Dehradun, INHS Asvini, INHS Sandhani.

\section{Inclusion and exclusion criteria of this study were}

- The diagnosis of ICP was based maternal pruritus in pregnancy with other causes of cholestasis excluded.

- Abnormal liver function tests-Elevation of serum aminotransferases (>30 U/l).

In this study, 50 women with ICP who satisfied the inclusion and exclusion criteria were started on UDCA therapy. They were started on UDCA medication as per the standard protocol using $300 \mathrm{mg} /$ twice a day.

A detailed history including maternal age, gravidity, parity, complications of earlier pregnancies, body mass index, and use of drugs, medical history and heredity of ICP was obtained. In subsequent follow-up visits the improvement in maternal pruritus, LFT levels side-effects of UDCA if any were also recorded along with foetal ultrasound reports.

\section{Maternal and foetal outcomes}

Gestational age at induction of labour, duration of labour and obstetrical managements, Apgar scores (1, $10 \mathrm{~min}$ ), birth weight were noted in this study.

\section{Statistical analysis}

The statistical analysis of the data thus collected is done by observational method of data analysis and computed in results respectively.

\section{RESULTS}

The patient's characteristics as collected during the study period was analysed and tabulated as shown in Table 1.
Table 1: The patient characteristics.

\begin{tabular}{|ll|l|}
\hline Variable & Range & Mean \\
\hline Age in years & $20-30$ & 26 \\
\hline Onset of pruritus (GA in weeks & $26-34$ & 32 \\
\hline ICP diagnosis at (weeks) & $28-36$ & 33 \\
\hline Parity & G1-multi & G1 \\
\hline
\end{tabular}

The mean gestational age at the beginning of pruritus was 32 weeks in the entire group.

As shown in the Table 1, the onset of ICP was in the late second and third trimester of pregnancy.

The mean gestational age at the diagnosis was 33 weeks and predominantly seen in first order pregnant women (Primigravidas).

\section{Response to therapy}

The protocol-based therapy with UDCA was started for all the patients in this study and the dose of UDCA was $300 \mathrm{mg} /$ twice a day as supported by the recent studies. The findings are shown below in Table 2 .

Table 2: Response to UDCA therapy.

\begin{tabular}{|ll|}
\hline Parameters & Improvement \\
\hline Pruritus & $50(100 \%)$ \\
\hline LFTs & $48(96 \%)$ \\
\hline Side effects & Nil \\
\hline
\end{tabular}

All women (100\%) in this study showed improvement in the pruritus during the subsequent Antenatal visits while on UDCA therapy. None of the patients showed any major adverse effects and intolerance to UDCA as shown in the Table 2. If this study.

\section{Liver function tests}

As shown in Table 2 lFTs begun to decrease after starting the medication.

On every visit to the antenatal clinic, LFTs were repeated, preferably on a weekly basis. There was a declining trend in the values of serum transaminases with near normal values in $48(96 \%)$ of women and in 2 cases the LFTs remained static but just above the normal levels.

Table 3: The obstetrics outcome in the study group.

\begin{tabular}{|ll|}
\hline Outcomes & Number of cases \\
\hline Normal delivery & $45(90 \%)$ \\
\hline LSCS & $5(10 \%)$ \\
\hline Gestational age at delivery & $37-38$ weeks \\
\hline NICU admission & Nil \\
\hline
\end{tabular}




\section{Maternal -foetal outcomes}

The obstetrics outcomes in this study were followed closely and analysed as shown in Table 3.

Obstetric and neonatal characteristics are presented in Table 3.

All patients in this study group were induced at 37 completed weeks of gestation with standard induction protocol.

Mothers on UDCA delivered at the mean gestational age of 37 completed weeks.

A total $45(90 \%)$ of women achieved successful normal delivery and in 5 cases LSCS was done in view of nonprogress of labor (3), cephalopelvic disproportion (2) cases respectively.

No adverse outcomes like postpartum hemorrhage, respiratory distress syndrome, still birth was observed in the present study which adds to the strength of present study.

Thus, the timing of delivery should be proper to reduce the ICP associated stillbirths at the same time not increasing the risks of prematurity.

\section{DISCUSSION}

UDCA is a very effective drug in the therapy of ICP. Studies conducted by Diaferia et al, supported the use of UDCA at dose of $600 \mathrm{mg} /$ day as in this study. ${ }^{13}$

Its use has been associated with lower frequency of fetal distress and reduced aminotransferase in all treated women with a significant reduction of pruritus. The prospective study of Palma et al, supports the close antenatal monitoring of pregnancies affected by ICP. ${ }^{14}$

In the present study also, UDCA was effective in reducing pruritus and improving liver function test results in patients with ICP.

In this study levels of aminotransferases begun to decrease after starting the UDCA therapy.

According to the latest Studies UDCA treatment should be recommended for women with ICP and elective delivery at 37 weeks in addition to monitoring fetal wellbeing can significantly reduce the stillbirth rate without increasing caesarean section rate. ${ }^{15-17}$

Mazzella et al, reported no adverse reactions of UDCA $(1.5-2 \mathrm{~g} / \mathrm{d})$ and showed UDCA therapy. ${ }^{18}$ Improves both biochemical and clinical parameters of cholestasis and is safe for the fetus.
UDCA was beneficial in ICP patients in terms of improving maternal pruritus, liver tests and also the final outcome of pregnancy. UDCA was also well tolerated and no adverse side effects were detected neither in mothers nor newborns followed up for 3 months after birth. Zapata et al, concluded that UDCA was well tolerated by pregnant women and no adverse effects were detected in his study comparable to the present study. ${ }^{19}$

In this study there were no preterm deliveries $(<37$ weeks) and associated NICU admissions. The recent study of Puljic et al, demonstrated that delivery at 36 weeks' gestation reduced the perinatal mortality risk as compared with expectant management. ${ }^{20}$ They concluded timing of delivery has to take into account both the reduction in stillbirth risk balanced with the morbidities associated with preterm delivery.

ICP increases the risk of respiratory distress syndrome in the new born. Chappell et al, also reported planned delivery not to increase caesarean section rate significantly in patients with ICP. ${ }^{21}$ As shown by Zecca et al, there were no stillbirths in the present study. ${ }^{22}$

Newborns in all the improved maternal and foetal outcomes supports the increased awareness of the disease, experienced management and protocol-based therapy had a good Apgar score of > 7, 9 at 1 and 5 minutes respectively. Rioseco et al study suggests that induction of labour may reduce intrauterine fetal death compared with expectantly management and is thus beneficial. ${ }^{22}$ The improved maternal and foetal outcomes supports the increased awareness of the disease, experienced management and protocol-based therapy.

\section{CONCLUSION}

UDCA protocol-based therapy reduces maternal liver function tests, is well tolerated by pregnant women and without any adverse maternal, fetal or neonatal side effects. UDCA is effective and safe in the treatment of pregnant women with ICP.

Funding: No funding sources

Conflict of interest: None declared

Ethical approval: The study was approved by the Institutional Ethics Committee

\section{REFERENCES}

1. Diken Z, Usta IM, Nassar AH. A clinical approach to intrahepatic cholestasis of pregnancy. Am J Perinatol. 2014;31:1-8.

2. Lammert F, Marschall HU, Glantz A, Matern S. Intrahepatic cholestasis of pregnancy: molecular pathogenesis, diagnosis and management. J Hepatol. 2000;33:1012-21.

3. Reyes H. Review: intrahepatic cholestasis. A puzzling disorder of pregnancy. J Gastroenterol Hepatol. 1997;12:211-6. 
4. Dixon PH, Wadsworth CA, Chambers J, Donnelly J, Cooley S, Buckley R, et al. A comprehensive analysis of common genetic variation around six candidate loci for intrahepatic cholestasis of pregnancy. Am J Gastroenterol. 2014;109(1):76-84.

5. Mackillop L, Williamson C. Liver disease in pregnancy. Postgrad Med J. 2010;86:160-4.

6. Saleh MM, Abdo KR. Intrahepatic cholestasis of pregnancy: review of the literature and evaluation of current evidence. J Womens Health (Larchmt). 2007;16:833-41.

7. Pevtichev S, Dimitrova V. Intrahepatic cholestasis of pregnancy. Akush Ginekol (Sofiia). 2013;52:35-41.

8. Glantz A, Marschall HU, Lammert F, Mattsson LÅ. Intrahepatic cholestasis of pregnancy: a randomized controlled trial comparing dexametasone and ursodeoxycholic acid. Hepatol. 2005;42:1399-405.

9. Lorente S, Montoro MA. Cholestasis of pregnancy. Gastroenterol Hepatol. 2007;30:541-7.

10. Roncaglia N, Locatelli A, Arreghini A, Assi F, Cameroni I, Pezzullo JC, et al. A randomized controlled trial of ursodeoxycholic acid and Sadenosyl-1-methionine in the treatment of gestational cholestasis. BJOG. 2004;111:17-21.

11. European Association for the Study of the Liver. Clinical practice guidelines: management of cholestatic liver diseases. J Hepatol. 2009;51(2):23767.

12. Diaferia A, Nicastri $P$, Tartagni $M$, Loizzi $P$, Iacovizzi C, Di Leo A. Ursodeoxycholic acid therapy in pregnant women with cholestasis. Int $\mathrm{J}$ Gynecol Obstet. 1996;52:133-40.

13. Palma J, Reyes H, Ribalta J, Iglesias J, Gonzalez M, Hernandez I et al. Effects of ursodeoxycholic acid in patients with intrahepatic cholestasis of pregnancy. Hepatol. 1992;15:1043-7.

14. Palma J, Reyes H, Ribalta J, Hernandez I, Sandoval $\mathrm{L}$, Almura R, et al. Ursodeoxycholic acid in the treatment of cholestasis of pregnancy: a randomised, double-blind study controlled with placebo. J Hepatol. 1997;27:1022-8.

15. Grand'Maison S, Durand M, Mahone M. The effects of ursodeoxycholic acid treatment for intrahepatic cholestasis of pregnancy on maternal and fetal outcomes: a meta-analysis including non-randomized studies. J Obstet Gynaecol Can. 2014;36:632-41.

16. Bacq Y, Sentilhes L, Reyes HB, Glantz A, Kondrackiene $\mathrm{J}$, Binder $\mathrm{T}$, et al. Efficacy of ursodeoxycholic acid in treating intrahepatic cholestasis of pregnancy: a meta-analysis. Gastroenterol. 2012;143:1492-501.

17. Mazzella G, Rizzo N, Azzaroli F, Simoni P, Bovicelli L, Miracolo A, et al. Ursodeoxycholic acid administration in patients with cholestasis of pregnancy: effects on primary bile acids in babies and mothers. Hepatol. 2001;33:504-8.

18. Zapata R, Sandoval L, Palma J, Hernandez I, Ribalta $\mathrm{J}$, Rayes $\mathrm{H}$, et al. Ursodeoxycholic acid in the treatment of intrahepatic cholestasis of pregnancy. A 12-year experience. Liver Int. 2005;25:548-54.

19. Puljic A, Kim E, Page J, Esakoff T, Shaffer B, LaCoursiere D, et al. The risk of infant and fetal death by each additional week of expectant management in intrahepatic cholestasis of pregnancy by gestational age. Am J Obstet Gyn. 2015;212:667.

20. Chappell LC, Gurung V, Seed PT. Ursodeoxycholic acid versus placebo, and early term delivery versus expectant management, in women with intrahepatic cholestasis of pregnancy: semifactorial randomised clinical trial. BMJ. 2012;344:e3799.

21. De Luca D, Marras M, Caruso A, Bernardini T, Romagnoli C. Intrahepatic cholestasis of pregnancy and neonatal respiratory distress syndrome. Pediatr. 2006;117:1669-72.

22. Rioseco AJ, Ivankovic MB, Manzur A, Hamed F, Kato SR, Parer JT, et al. Intrahepatic cholestasis of pregnancy: a retrospective case-control study of perinatal outcome. Am J Obstet Gynecol. 1994;170:890-5.

Cite this article as: Pitale DI, Jadhav SN.

Effectiveness of ursodeoxycholic acid therapy in intrahepatic cholestatsis of pregnancy. Int J Reprod Contracept Obstet Gynecol 2020;9:1069-72. 